e il primo contatto interno a

$$
3^{\mathrm{h}} \quad 9^{\mathrm{m}} 34^{\mathrm{s}} \cdot 79
$$

Il Professore Millosevich notò il primo contatto esterno a

$$
2^{\mathrm{h}} 49^{\mathrm{m}} 4^{8}: \mathrm{I} 4
$$

e il primo interno a

$$
3^{\mathrm{h}} \quad 9^{\mathrm{m}} 29^{\mathrm{s}} \cdot 34
$$

al momento della formazione della goccia nera e

$$
3^{\mathrm{h}} 10^{\mathrm{m}} 10^{\mathrm{s}} \mathrm{I} 4
$$

al momento della scomparsa della goccia međiesima.
Poco dopo avvenuto il primo contatto esterno, il Prof. Milloserich avverti per il primo la presenza dell atmosfera di Venere, che fu poi reduta anche da me e dall' assistente Sr Chistoni.

Allo spettroscopio fu pure notato, come al 1874 , l'effetto prodotto dalla detta atmosfera nello spettro del sole. Il disco del pianeta era del tutto nero, come la banda da esso prodotta nello spettroscopio. Fuori del bordo del sole e fuori della cromosfern solare il pianeta non era visibile. Misurai allo spettroscopio sulla riga $C$ il diametro di Venere, e il medio dei passaggi registrati al cronografo, $\mathrm{mi}$ ha dato $p \in \mathbf{r}$ il diametro del pianeta il ralore di 67.25 .

Roma I 882 Dic. 7 .

\title{
Beobachtung des Venusdurchganges auf der Sternwarte München.
}

Kurz vor Eintritt der Venus in die Sonnenscheibe hellte noch der vorher vollständig mit Wolken bedeckte Himmel auf und gestattete die Verfolgung des seltenen Phänomens. Während ich an einem Fraunhofer mit 43 Linien Oeffnung beobachtete, stand dem Gehilfen der Sternwarte, Herrn List, ein Fraunhofer mit 34 Linien Oeffnung zu Gebote. Es ergab sich in M. Z. München

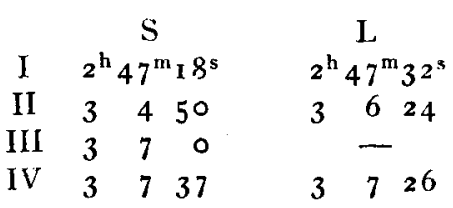

Dabei bedeutet I den äusseren Eintritt, II den geometrischen inneren Contact; bei III erschien ziemlich plötzlich eine feine Lichtlinie, welche den Venusrand ron dem schwarzen Rande trennte, während bei IV die Losreissung des 'Tropfens vollendet schien. Bei der geringen Höhe der Sonne und der ausserordentlich grossen Unruhe des Sonnenbildes darf selbstverständlich den beobachteten Zeitmomenten nur eine sehr geringe Zuverlässigkeit zugeschrieben werden. Der äussere Eintritt geschah überdies bei nicht völlig freiem Himmel und beruht seine Notirung auf einer unsicheren Schätzung.

Sehr erschwerend hat die grosse Unruhe der Bilder auf die unter II, III und IV aufgeführten Beobachtungen gewirkt. In der That war die Figur der Venus zeitweilig so verzerrt, dass das Phänomen gar keine bestimmte Erscheinungsform annahm. Im Allgemeinen habe ich den Eindruck empfangen, dass das wichtigste Moment IV wahrscheinlich $z u$ spät beobachtet worden ist. Im Moment III, bei dem übrigens möglicherweise $6^{\mathrm{m}_{0}}$ statt $7^{\mathrm{m}_{0}}$ s gelesen werden muss, tauchte ziemlich plötzlich eine helle
Lichtlinie an dem dem Sonnenrand näheren Venusrand auf. Im Augenblicke der Notirung, da diese Linie nicht wieder verschwand, sondern eher breiter wurde, glaubte ich den Beginn des Zerreissens des Tropfens zu beobachten; später bin ich an dieser Auffassung zweifelhaft geworden und bin ich gegenwärtig eher geneigt, auch diese Erscheinung als eine Wirkung der Venusatmosphäre zu betrachten.

Was die Wirkung dieser letzteren betrifft, so bin ich durch die Deutlichkeit ihres Hervortretens iberrascht gewesen; ich hatte in Jahre 1874 auch nicht entfernt Aehnliches bemerkt, was allerdings durch die Umstände, unter denen ich damals den Austritt beobachten konnte, nämlich nach Absolvirung mehrstündiger anstrengender heliometrischer Messungen, völlig erklärlich ist.

Bereits um $2^{\mathrm{h}} 5^{6^{\mathrm{m}}}$ sah ich die ganze Venusscheibe auch ausserhalb der Sonne sich als dunkle Fläche projiciren. Um $2^{\mathrm{h}} 5^{8^{\mathrm{m}}}$ fing sich ein Lichtbogen von $90^{\circ}$ Länge längs des südlichen ausserhalb der Sonne befindlichen Venusrandes zu bilden an. Um $2^{\mathrm{h}} 5^{8^{\mathrm{m}}} 4 \mathrm{o}^{\mathrm{s}}$ war der ganze äussere Venusrand durch einen hellen feinen Lichtbogen kenntlich, der an Intensität zunahm und um $3^{\mathrm{h}} 1^{\mathrm{m}}$ in geradezu überraschender Deutlichkeit sich darstellte. Die Schwankungen in der Deutlichkeit und Intensität dieses Lichtbogens dürften nun freilich im Wesentlichen von dem momentanen Zustand der Atmosphäre abhängen. Ich zweifle aber nicht, dass es im Allgemeinen sehr deutliche Wirkungen einer Venusatmosphäre waren, welche sich in den obigen, unter den erwähnten für die Zeitnotirungen so überaus ungünstigen Verhältnissen angestellten Beobachtungen ausgesprochen haben.

Bogenhausen 1882 December 7 .

\section{In halt:}

Zu Nr. 2478. Wilhelm Schur. Bestimmung der Masse des Planeten Jupiter aus Heliometer-Beobachtungen der Abstände seiner Satelliten. 81 . F. F. Ful. Schmidt. Ortsbestimmung des grossen Cometen von 1882 zu $\Lambda$ then 85. - F. F. Ful. Sclimidt. Ueber die Gestalt des Schweifes des grossen Cometen von 1882. 89. - R: Wolf. Auszug aus einem Schreiben an den Herausgeber. 93. - A. N. Skinner. Meridian observation of the Great Comet of September 1882. 93. - P. Tacchini. Osservazione del passaggio di Venere I882 Dic. 6 a Roma. 93. - H. Seeliger. Beobachtung des Venusdurchgangs auf der Sternwarte in München. 95.

Geschlosen 1882 Dec. 20. Herauggeber: A. Krueger. Druck von C. F. Mohr. Expedition: Sternwarte in Kiel. 